\title{
Fish consumption and frying of fish in relation to type 2 diabetes incidence: a prospective cohort study of Swedish men
}

\author{
Alice Wallin ${ }^{1} \cdot$ Daniela Di Giuseppe $^{1} \cdot$ Nicola Orsini $^{1} \cdot$ Agneta Åkesson $^{1} \cdot$ \\ Nita G. Forouhi ${ }^{2}$ - Alicja Wolk ${ }^{1}$
}

Received: 25 August 2015 / Accepted: 6 December 2015 / Published online: 21 December 2015

(C) Springer-Verlag Berlin Heidelberg 2015

\begin{abstract}
Purpose Epidemiological evidence on the association between fish consumption and risk of type 2 diabetes is heterogeneous across geographical regions. Differences related to fish consumption pattern could possibly help explain the discrepancy between the findings. We therefore aimed to investigate the association between fish consumption (total, fried, specific fish items) and type 2 diabetes incidence, taking exposure to contaminants present in fish (polychlorinated biphenyls and methyl mercury) into consideration.

Methods The population-based Cohort of Swedish Men, including 35,583 men aged 45-79 years, was followed from 1998 to 2012. We estimated hazard ratios (HRs) with $95 \%$ confidence intervals (CIs) using Cox proportional hazards models.

Results During 15 years of follow-up, 3624 incident cases were identified. Total fish consumption ( $\geq 4$ servings/week vs. $<1$ serving/week) was not associated with type 2 diabetes in multivariable-adjusted analysis (HR 1.00; $95 \%$ CI 0.85-1.18); however, a statistically non-significant inverse association was observed after adjustment for dietary
\end{abstract}

Electronic supplementary material The online version of this article (doi:10.1007/s00394-015-1132-6) contains supplementary material, which is available to authorized users.

Alice Wallin

alice.wallin@ki.se

1 Unit of Nutritional Epidemiology, Institute of Environmental Medicine, Karolinska Institutet, Box 210, 17177 Stockholm, Sweden

2 Medical Research Council Epidemiology Unit, Institute of Metabolic Science, University of Cambridge School of Clinical Medicine, Cambridge, UK contaminant exposures (HR 0.79; $95 \%$ CI $0.60-1.04$ ). Fried fish ( $\geq 6$ servings/month vs. $\leq 1$ servings/month) and shellfish consumption ( $\geq 1$ serving/week vs. never/seldom) were associated with HRs of 1.14 (95\% CI 1.03-1.31) and 1.21 (95\% CI 1.07-1.36), respectively.

Conclusions We observed no overall association between total fish consumption and type 2 diabetes. The results indicated that dietary contaminants in fish may influence the relationship. Fried fish and shellfish consumption were associated with higher type 2 diabetes incidence. These findings suggest that more specific advice on fish species sub-types (varying in contamination) and preparation methods may be warranted.

Keywords Fish · Fried foods · Polychlorinated biphenyls · Methyl mercury · Type 2 diabetes .

Cohort study

\section{Introduction}

Type 2 diabetes is a growing public health burden, and the prevalence has reached epidemic proportions globally [1]. Fish consumption has been hypothesized to be protective for type 2 diabetes given the beneficial effects seen on cardiovascular risk factors and cardiovascular diseases (CVD) [2]. Results from epidemiological studies on fish consumption in relation to risk of developing diabetes have, however, been largely inconsistent and inconclusive [3-16]. Several meta-analyses have highlighted the heterogeneity of results and brought attention to potential geographical differences, with inverse associations observed in studies conducted in Asia, overall null associations in European populations and higher risks in US populations [1719]. Differences in fish consumption pattern potentially 
determined by geographical location (e.g. types of fish consumed, preparation methods and degree of contamination) could possibly help explain the discrepancy between the findings, but previous studies on fish consumption in relation to type 2 diabetes risk have not fully accounted for such factors. Frying of fish could potentially be of importance because of formation of advanced glycation end products (AGEs) [20], mutagenic compounds [21], changes in fatty acid composition or increased energy-density. Further, studies on CVD where fried fish was distinguished from non-fried fish have indicated that the associations may vary by preparation method [22-26]. Contaminants present in fish may also be of importance. Fish is the main source of dietary exposure to both persistent organic pollutants such as polychlorinated biphenyls (PCBs) [27] and methyl mercury $(\mathrm{MeHg})$ [28], which have been associated with type 2 diabetes [29, 30]. We therefore aimed to investigate the association of total fish consumption, fried fish and specific fish items with incidence of type 2 diabetes in a large population-based prospective study, taking exposure to contaminants present in fish into consideration.

\section{Subjects and methods}

\section{Study population}

The Cohort of Swedish Men is a prospective populationbased study initiated in the autumn of 1997. All men born between 1918 and 1952 (45-79 years of age) and residing in Örebro and Västmanland counties of central Sweden received an invitation to participate in the study together with a questionnaire on diet and other lifestyle factors. A total of 48,850 men returned the questionnaire (response rate $49 \%$ ). In 2008, an extended questionnaire on health, including a question on diabetes status, was sent out (response rate $70 \%$ ). The cohort is representative of Swedish men aged 45-79 years in terms of age distribution, educational level and prevalence of overweight [31]. The study was approved by the Regional Ethical Review Board at Karolinska Institutet (Stockholm, Sweden), and return of the completed questionnaire was considered to imply informed consent.

From the baseline population, we excluded men with incorrect or incomplete national identification number $(n=205)$, those who returned an inadequately completed questionnaire $(n=92)$ and those who died $(n=55)$ or had a cancer diagnosis (not including non-melanoma skin cancer; $n=2592$ ) before 1 January 1998 . We further excluded prevalent cases of diabetes based on registry data from the Swedish National Diabetes Register (NDR) and the Swedish National Patient Register (NPR) and baseline self-reports $(n=3404)$. Moreover, we excluded those who reported a diabetes diagnosis in the 2008 questionnaire that could not be confirmed by registry data $(n=67)$ and those who were registered with non-type 2 diabetes during the follow-up period $(n=200)$. In addition, we excluded participants with a history of CVD (myocardial infarction, angina and stroke; $n=4537$ ), because they may have changed their dietary habits after diagnosis and for comparability with other studies. We also excluded those with implausible values for total energy intake $( \pm 3$ SD from the log-transformed mean energy intake; $n=398$ ), those who did not fill in any of the questions in the fish intake section of the questionnaire $(n=239)$ and those with missing information on frying frequency $(n=1478)$. After these exclusions, 35,583 men remained for analysis.

\section{Assessment of diet and covariates}

Dietary intake was assessed at baseline using a 96-item food frequency questionnaire (FFQ) in which participants were asked to report their average frequency of consumption of various foods over the previous year. Eight predefined frequency categories were used (never/seldom; 1-3 times/month; 1-2 times/week; 3-4 times/week; 5-6 times/week; 1 time/day; 2 times/day; $\geq 3$ times per day). With regard to fish consumption, participants were asked to report consumption frequency of three different finfish types (herring/mackerel, salmon/whitefish/char, cod/saithe/ fish fingers) as well as of shellfish (shrimp/crayfish, etc.). Partial non-response in the fish intake section was assumed to imply never/seldom. This was based on a study among Swedish men and women showing that $82 \%$ of omitted answers on fish consumption corresponded to non-consumption [32]. The midpoint of the chosen frequency range on each item was converted to servings/week and summed to obtain an estimate of total fish consumption (sum of herring/mackerel, salmon/whitefish/char and cod/saithe/fish fingers). Moreover, participants were specifically asked to report monthly frequency of fried fish consumption in a separate open section of the questionnaire (without predefined frequency categories).

Calculation of total energy intake was based on agespecific $(\leq 52,53-61,62-69, \geq 70$ years $)$ portion sizes that were derived from two 1-week weighted dietary records completed by a random sample of 152 men. Food composition values were obtained from the Swedish National Food Agency Database [33].

The validity of the FFQ has been assessed in a random sample of 248 men aged $40-74$ years. The Spearman correlation coefficients between FFQ-based estimates and the mean of 14 repeated 24-h recall interviews during a 1-year period were 0.65 for macronutrients and 0.62 for micronutrients [34]. In a validation study of the FFQ among 129 women of the same age and from the same study area, the 
correlation between the FFQ-based estimates and those from four 1-week weighted diet records ranged from 0.4 to 0.6 for fish and shellfish items (A. Wolk, unpublished data).

To account for overall diet in the analyses and reduce the number of covariates included in the models, we used a quality score instead of several single food groups. As an overall dietary quality score, we calculated a DASH (Dietary Approaches to Stop Hypertension) diet component score using the score proposed by Fung et al. [35], which ranks participants according to their intake of eight dietary components. Higher scores were given for high intake of fruits, vegetables, nuts and legumes, low-fat dairy and whole grains, and for low intake of sodium, sweetened beverages, and red and processed meats.

For FFQ-based estimation of dietary exposure to PCBs and $\mathrm{MeHg}$, we have used recipe-based databases created for the FFQ. The PCB database, described in detail elsewhere [27], was based on the PCB congener 153, the most abundant congener in Swedish food and an excellent indicator for total PCB in food, blood and adipose tissue [36, 37]. Concentrations of $\mathrm{PCBs}$ and $\mathrm{MeHg}$ in foods were obtained from the Swedish National Food Agency and the Swedish Environmental Protection Agency, and dietary exposure estimates were calculated using the consumption frequency and age-specific portion sizes of the respective foods. For each food item in the FFQ, the contaminant calculations were based on a larger number of specific food types according to the distribution of consumption in the population. The exposure estimates were adjusted to the mean energy intake in the cohort using the residual method [38]. Fish and shellfish contributed to about $2 / 3$ of the total dietary PCB exposure in the cohort. Estimation of dietary $\mathrm{MeHg}$ exposure was based on fish and shellfish only as it is practically the only source of dietary $\mathrm{MeHg}$, with lean fish species contributing to about $50 \%$ in the cohort. The long-term dietary PCB exposure estimates showed reasonable validity against the sum of 6 PCB congeners measured in serum [correlation $(r), 0.48 ; p<0.001$ ] in a sample of 201 Swedish women [27]. FFQ-estimated fish consumption has been shown to correlate well with hair mercury concentrations in a Swedish population $(r=0.75$; $p<0.001)$ [28].

Information on height, body weight, education, alcohol consumption, smoking habits and physical activity was collected via the baseline questionnaire. Body mass index (BMI) was calculated by dividing weight (in $\mathrm{kg}$ ) by the square of height (in m). For total physical activity, the reported daily time spent in specific activities (work/occupational activity, home/household work, walking/bicycling, watching TV/reading, exercising and sleeping) was multiplied by their typical energy expenditure requirements [expressed in metabolic equivalents (METs)] and summed to create a MET-h/day score [39].

\section{Case ascertainment and follow-up of the cohort}

Study participants were followed from 1 January 1998 to 31 December 2012. Incident cases of type 2 diabetes were identified by linkage of the study cohort with the Swedish National Diabetes Register (NDR) and the Swedish National Patient Register (NPR). The NDR was initiated in 1996 and contains clinical information from patient visits reported at least once per year, and it also includes retrospective recording of diabetes onset year. Based on expected diabetes prevalence, the coverage of NDR is estimated to be nearly complete in the study area [40]. In validation against the Swedish Prescribed Drug Register, over $90 \%$ of individuals aged $50-80$ years on diabetes medication were covered by NDR [40]. Reporting to the NPR is mandatory, and it includes information on main and secondary diagnoses for all inpatient care visits in Sweden since 1987 and also outpatient visits from both private and public caregivers since 2001. ICD-10 code E11 was used to identify cases of type 2 diabetes. The first available date in either of the two registers was considered as the diagnosis date. Dates of death were obtained from the Swedish Death Register.

\section{Statistical analysis}

We used Cox proportional hazards regression models to estimate hazard ratios (HRs) with $95 \%$ confidence intervals (CIs) for the associations between total fish consumption (sum of three finfish items: herring/mackerel, salmon/ whitefish/char and cod/saithe/fish fingers), fried fish consumption, specific fish items and incidence of type 2 diabetes. Total fish was categorized in five groups $(<1,1-<2$, $2-<3,3-<4$ and $\geq 4$ servings/week). Of note, the recommended fish consumption in Sweden, 2-3 servings/week, is represented in the mid-category. Overall, the categorization reflects the consumption pattern in the study population. As the number of participants reporting no fish consumption was small $(n=911 ; 2.6 \%)$, we also included low consumers in the reference category. Nevertheless, to test the robustness of our results, we performed additional analyses with only non-consumers of fish as the reference group. For the categorization of fried fish consumption, we used approximate quintiles $(\leq 1,2,3-4,5, \geq 6$ servings/ month). Individual fish items were categorized into three groups (never/seldom, 1-3 servings/month, $\geq 1$ serving/ week) with the two lower directly corresponding to the two lowest predefined frequency categories in the FFQ and the third combining the remaining six higher response categories. Age was used as underlying timescale in all analyses. Each participant contributed person-time from 1 January 1998 until the date of type 2 diabetes diagnosis, death or end of follow-up, whichever came first. 
The primary multivariable analyses were adjusted for BMI (kg/m²; <20, 20-24.9, 25-29.9, $\geq 30)$, physical activity (MET-h/day; quartiles), education (primary school, high school, university), cigarette smoking (never, former, current $\leq 10$ cigarettes/day or $>10$ cigarettes/day), total energy intake (kcal/day; quintiles), intake of alcohol (g/day; quartiles) and DASH diet component score (based on intake of fruits, vegetables, nuts and legumes, low-fat dairy, whole grains, sodium, sweetened beverages, and red and processed meats; quartiles). Analyses of specific fish items were mutually adjusted for the other three types. Multivariable model 2 was further adjusted for dietary exposure to PCBs (ng/day, quintiles) and $\mathrm{MeHg}$ ( $\mu \mathrm{g} /$ day, quintiles). Missing values for any covariates were treated as a separate category in the model. The Schoenfeld residual test indicated no violation of the proportional hazard assumption. Tests for linear trend were performed by assigning each participant the median value in their respective exposure category and modelling this as a continuous variable. We further examined the dose-response relationship between fried fish consumption and HR of type 2 diabetes using restricted cubic splines with knots at 10, 50 and $90 \%$ of the distribution. A $p$ value for nonlinearity was obtained by testing the null hypothesis that the coefficient of the second spline is equal to 0 .

In a sensitivity analysis, we excluded men who reported any use of fish oil supplements ( $n=1649 ; 4.6 \%$ of the study population).

Statistical analyses were carried out using Stata (version 13.0 ), and $p$ values $\leq 0.05$ were considered statistically significant.

\section{Results}

During 15 years of follow-up (467,961 person-years), 3624 incident cases of type 2 diabetes were identified. The mean $( \pm \mathrm{SD})$ number of servings consumed per week was $0.6( \pm 0.8)$ of herring/mackerel, $0.4( \pm 0.9)$ of salmon/ whitefish/char, $0.9( \pm 1.0)$ of $\mathrm{cod} / \mathrm{saithe} / \mathrm{fish}$ fingers and $0.4( \pm 0.6)$ of shellfish. The mean frequency of consuming fried fish was $3.2( \pm 2.9)$ times/month. The mean energyadjusted dietary exposure to PCBs was $274( \pm 191) \mathrm{ng} / \mathrm{day}$ and to $\mathrm{MeHg} 1.5( \pm 1.3) \mu \mathrm{g} / \mathrm{day}$.

Age-standardized baseline characteristics by total fish consumption are presented in Table 1. On average, men
Table 1 Age-standardized baseline characteristics of 35,583 participants of the Cohort of Swedish Men, by total fish consumption

\begin{tabular}{llllll}
\hline & \multicolumn{4}{l}{ Categories of total fish consumption, servings/week (median) } \\
\cline { 2 - 6 } & $<1(0.9)$ & $1-<2(1.4)$ & $2-<3(2.4)$ & $3-<4(3.5)$ & $\geq 4(5.0)$ \\
\hline No. of participants & 10,867 & 13,458 & 6292 & 3367 & 1599 \\
Age (mean \pm SD, years) & $58.8 \pm 9.4$ & $59.1 \pm 9.2$ & $58.7 \pm 9.2$ & $62.2 \pm 9.6$ & $62.7 \pm 9.7$ \\
BMI (mean, kg/m ${ }^{2}$ ) & 26 & 26 & 25 & 26 & 26 \\
Total physical activity (mean, MET-h/day) & 42 & 42 & 41 & 42 & 42 \\
University education (\%) & 14 & 18 & 23 & 21 & 22 \\
Current smokers (\%) & 27 & 24 & 20 & 25 & 27 \\
Alcohol (mean, g/day) & 15 & 15 & 16 & 17 & 21 \\
Energy intake (mean, kcal/day) & 2529 & 2689 & 2861 & 2922 & 3263 \\
Fried fish (mean, servings/month) & 2.5 & 3.2 & 3.6 & 4.3 & 4.8 \\
Dietary components (mean, g/1000 kcal) & & & & & \\
Fruits & 63 & 68 & 76 & 75 & 80 \\
Vegetables & 45 & 53 & 60 & 61 & 68 \\
Nuts and legumes & 16 & 17 & 19 & 21 & 26 \\
Whole grains & 78 & 81 & 82 & 85 & 79 \\
Low-fat dairy & 116 & 114 & 113 & 108 & 101 \\
Sodium & 1.4 & 1.4 & 1.5 & 1.5 & 1.6 \\
Red and processed meats & 38 & 40 & 40 & 40 & 45 \\
Sweetened beverages & 77 & 72 & 62 & 65 & 62 \\
Dietary PCB exposure (mean, ng/day) & 162 & 241 & 314 & 442 & 776 \\
Dietary MeHg exposure (mean, $\mu \mathrm{g} / \mathrm{day})^{\mathrm{a}}$ & 0.7 & 1.4 & 1.9 & 2.4 & 4.1 \\
\hline & & & & & \\
\hline
\end{tabular}

Total fish is the sum of three finfish items: herring/mackerel, salmon/whitefish/char and cod/saithe/fish fingers; all variables except age are standardized to the age distribution of the study cohort

$B M I$ body mass index, $M E T$ - $h /$ day metabolic equivalent hours per day

${ }^{a}$ Adjusted to the mean energy intake in the cohort 
Table 2 Hazard ratios of type 2 diabetes by consumption of total fish, the Cohort of Swedish Men 1998-2012

\begin{tabular}{|c|c|c|c|c|c|c|}
\hline & \multicolumn{5}{|c|}{ Categories of total fish consumption, servings/week (median) } & \multirow[t]{2}{*}{$p$ for trend } \\
\hline & $<1(0.9)$ & $1-<2(1.4)$ & $2-<3(2.4)$ & $3-<4(3.5)$ & $\geq 4(5)$ & \\
\hline No. of cases & 1104 & 1363 & 609 & 378 & 170 & \\
\hline Person-years & 142,599 & 178,573 & 84,243 & 42,606 & 19,940 & \\
\hline Age-adjusted model & 1.00 (ref) & $0.97(0.90-1.05)$ & $0.93(0.84-1.03)$ & $1.06(0.94-1.19)$ & $1.01(0.86-1.19)$ & 0.67 \\
\hline 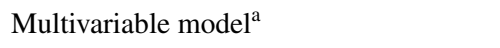 & 1.00 (ref) & $1.02(0.95-1.11)$ & $1.01(0.91-1.12)$ & $1.10(0.98-1.24)$ & $1.00(0.85-1.18)$ & 0.48 \\
\hline Multivariable model $2(+ \text { contaminants })^{b}$ & 1.00 (ref) & $0.95(0.84-1.07)$ & $0.86(0.72-1.03)$ & $0.89(0.71-1.12)$ & $0.79(0.60-1.04)$ & 0.13 \\
\hline
\end{tabular}

Total fish is the sum of three finfish items: herring/mackerel, salmon/whitefish/char and cod/saithe/fish fingers

Adjusted for attained age, body mass index $\left(\mathrm{kg} / \mathrm{m}^{2} ;<20,20-24.9,25-29.9, \geq 30\right)$, physical activity (metabolic equivalent hours per day; quartiles), education (primary school, high school, university), cigarette smoking (never, former, current $\leq 10$ cigarettes/day or $>10$ cigarettes/day), total energy intake (kcal/day; quintiles), intake of alcohol (g/day; quartiles) and DASH diet component score (based on intake of fruits, vegetables, nuts and legumes, low-fat dairy, whole grains, sodium, sweetened beverages, and red and processed meats; quartiles)

${ }^{a}$ Additionally adjusted for dietary exposure to polychlorinated biphenyls (ng/day, quintiles) and methyl mercury ( $\mu \mathrm{g} / \mathrm{day}$, quintiles)

who consumed more fish had a higher energy intake and a higher consumption per $1000 \mathrm{kcal}$ of red and processed meats, fruit, vegetables, and nuts and legumes and a lower consumption of low-fat dairy; they were also slightly older and more likely to have a university education.

Table 2 shows HRs with $95 \%$ CIs for type 2 diabetes by categories of total fish consumption. No association was observed in age-adjusted analyses or after multivariable adjustment for BMI, physical activity, education, cigarette smoking, alcohol and dietary factors. After further adjustment for contaminants, there was a statistically non-significant inverse association comparing those who consumed $\geq 4$ servings/week with those who consumed $<1$ serving/ week (HR 0.79; $95 \%$ CI 0.60-1.04). Similar results were observed when never/seldom consumers $(n=911)$ were used as the reference, both in the primary model (HR $0.94 ; 95 \%$ CI $0.73-1.21$ ) and in the contaminant-adjusted model (HR 0.75 ; $95 \%$ CI $0.52-1.07$ ). While dietary PCB and $\mathrm{MeHg}$ exposure had similar impact on the association between fish consumption and diabetes incidence, neither of them were significantly associated with type 2 diabetes incidence: HRs, 1.07 (95\% CI 0.90-1.26; $\left.p_{\text {trend }}=0.41\right)$ and 1.14 (95\% CI 0.95-1.36; $p_{\text {trend }}=0.06$ ) for PCB and $\mathrm{MeHg}$, respectively, comparing extreme quintiles.

Exclusion of the first 2 years of follow-up from the main analysis (305 cases diagnosed in 1998-1999) had little impact on the observed associations ( $\geq 4$ servings/week vs. $<1$ serving/week of total fish, model 1: HR 1.01; $95 \%$ CI 0.85-1.20; model 2: HR 0.79; $95 \%$ CI 0.59-1.05).

The Spearman correlation between total fish consumption and quintiles of dietary contaminant exposure was 0.77 for PCB and 0.70 for MeHg. To explore potential impact of the statistical adjustment of collinear variables, we examined the association between total fish consumption (categorized into $<1$ serving/week, 1-2 servings/week and $>2$ servings/week) and incidence of type 2 diabetes across strata of dietary PCB and MeHg exposures (median splits). Comparing men with a high fish consumption ( $>2$ servings per week) with those who consumed less than 1 weekly serving, the results were similar for men with dietary PCB exposure below the median (multivariable HR 0.91; $95 \%$ CI $0.69-1.22$ ) and above the median (multivariable HR 0.87; $95 \%$ CI 0.70-1.09). The corresponding HRs for men below and above the median dietary $\mathrm{MeHg}$ exposure were 1.02 (95\% CI 0.83-1.25) and 0.91 (95\% CI 0.75-1.10).

Fried fish consumption ( $\geq 6$ servings/month vs. $\leq 1$ serving/month) was associated with higher incidence of type 2 diabetes (HR 1.14; $95 \%$ CI 1.03-1.31) in the multivariable model (Table 3). Further adjustment for the contaminants did not markedly alter these results (HR 1.13; $95 \%$ CI $1.00-1.28)$. In the restricted cubic spline model, we found no evidence of a nonlinear association between fried fish consumption and incidence of type 2 diabetes $\left(p_{\text {nonlinearity }}=\right.$ 0.21 ). Each one serving/week increment in fried fish consumption was associated with an HR of $1.07(95 \% \mathrm{CI}$ 1.02-1.11).

No associations were observed for the three types of finfish separately (Supplementary table), whereas consumption of shellfish ( $\geq 1$ serving/week vs. never/seldom) was associated with higher type 2 diabetes incidence (HR 1.21; $95 \%$ CI 1.07-1.36) in the multivariable model (Table 4). Additional adjustment for dietary PCB and $\mathrm{MeHg}$ exposure resulted in slightly lower point estimates for all finfish types, but the associations remained non-significant. The association with shellfish was not markedly influenced (HR 1.19 ; $95 \%$ CI 1.04-1.35).

Exclusion of fish oil supplement users $(n=1649 ; 178$ cases) had little impact on the multivariable-adjusted estimates comparing the highest vs. lowest categories of consumption (total fish: HR 1.03; $95 \%$ CI 0.87-1.22, fried fish: HR 1.11; $95 \%$ CI 0.98-1.25, shellfish: HR 1.18; $95 \%$ CI 1.05-1.34). 
Table 3 Hazard ratios of type 2 diabetes by consumption of fried fish, the Cohort of Swedish Men 1998-2012

\begin{tabular}{|c|c|c|c|c|c|c|}
\hline & \multicolumn{5}{|c|}{ Categories of fried fish consumption, servings/month (median) } & \multirow[t]{2}{*}{$p$ for trend } \\
\hline & $\leq 1(0)$ & $2(2)$ & $3-4(4)$ & $5(5)$ & $\geq 6(8)$ & \\
\hline No. of cases & 779 & 624 & 1359 & 435 & 427 & \\
\hline Person-years & 118,221 & 85,359 & 165,762 & 50,258 & 48,361 & \\
\hline Age-adjusted model & 1.00 (ref) & $1.10(0.99-1.23)$ & $1.20(1.10-1.31)$ & $1.29(1.15-1.45)$ & $1.25(1.11-1.41)$ & $<0.001$ \\
\hline Multivariable model $^{\mathrm{a}}$ & 1.00 (ref) & $1.08(0.97-1.20)$ & $1.15(1.05-1.26)$ & $1.16(1.03-1.31)$ & $1.14(1.03-1.31)$ & 0.004 \\
\hline Multivariable model $2(+ \text { contaminants })^{\mathrm{b}}$ & 1.00 (ref) & $1.08(0.97-1.21)$ & $1.15(1.05-1.26)$ & $1.16(1.03-1.30)$ & $1.13(1.00-1.28)$ & 0.01 \\
\hline
\end{tabular}

${ }^{a}$ Adjusted for attained age, body mass index $\left(\mathrm{kg} / \mathrm{m}^{2} ;<20,20-24.9,25-29.9, \geq 30\right.$ ), physical activity (metabolic equivalent hours per day; quartiles), education (primary school, high school, university), cigarette smoking (never, former, current $\leq 10$ cigarettes/day or $>10$ cigarettes/day), total energy intake (kcal/day; quintiles), intake of alcohol (g/day; quartiles) and DASH diet component score (based on intake of fruits, vegetables, nuts and legumes, low-fat dairy, whole grains, sodium, sweetened beverages, and red and processed meats; quartiles)

b Additionally adjusted for dietary exposure to polychlorinated biphenyls (ng/day, quintiles) and methyl mercury ( $\mu \mathrm{g} / \mathrm{day}$, quintiles)

Table 4 Hazard ratios of type 2 diabetes by consumption of shellfish, the Cohort of Swedish Men 1998-2012

\begin{tabular}{|c|c|c|c|c|}
\hline & \multicolumn{3}{|c|}{ Categories of shellfish consumption (median) } & \multirow[t]{2}{*}{$p$ for trend } \\
\hline & Never/seldom & $1-3$ servings/month & $\geq 1$ serving/week $(1.5)$ & \\
\hline No. of cases & 1006 & 2161 & 457 & \\
\hline Person-years & 128,943 & 286,299 & 52,719 & \\
\hline Age-adjusted model & 1.00 (ref) & $1.05(0.97-1.13)$ & $1.21(1.08-1.35)$ & 0.001 \\
\hline Multivariable model $^{\mathrm{a}}$ & 1.00 (ref) & $1.12(1.03-1.22)$ & $1.21(1.07-1.36)$ & 0.003 \\
\hline Multivariable model $2(+ \text { contaminants })^{\mathrm{b}}$ & $1.00(\mathrm{ref})$ & $1.12(1.03-1.22)$ & $1.19(1.04-1.35)$ & 0.01 \\
\hline
\end{tabular}

a Adjusted for attained age, body mass index $\left(\mathrm{kg} / \mathrm{m}^{2} ;<20,20-24.9,25-29.9, \geq 30\right.$ ), physical activity (metabolic equivalent hours per day; quartiles), education (primary school, high school, university), cigarette smoking (never, former, current $\leq 10$ cigarettes/day or $>10$ cigarettes/day), total energy intake (kcal/day; quintiles), intake of alcohol (g/day; quartiles), DASH diet component score (based on intake of fruits, vegetables, nuts and legumes, low-fat dairy, whole grains, sodium, sweetened beverages, and red and processed meats; quartiles) and consumption of the three finfish types (herring/mackerel, salmon/whitefish/char and cod/saithe/fish fingers; never/seldom, 1-3 servings/month, $\geq 1$ serving/week)

${ }^{\mathrm{b}}$ Additionally adjusted for dietary exposure to polychlorinated biphenyls (ng/day, quintiles) and methyl mercury ( $\mu \mathrm{g} / \mathrm{day}$, quintiles)

\section{Discussion}

In this large population-based cohort of men, there was no overall association between total fish consumption and incidence of type 2 diabetes. However, there was a statistically non-significant inverse association after taking dietary exposure to contaminants present in fish (PCBs and $\mathrm{MeHg}$ ) into account. Fried fish consumption and shellfish consumption were associated with higher incidence of type 2 diabetes, and these associations were not markedly influenced by additional adjustment for contaminants.

Previous cohort studies investigating total fish consumption in relation to risk of type 2 diabetes have provided conflicting results. Inverse associations were observed in three studies $[6,12,13]$, no association in eight $[3,4,9,10,14-$ $16,41]$ and direct associations in three studies [5, 7, 11]. Shellfish consumption has been specifically addressed in six studies, also providing heterogeneous results suggesting inverse [13], direct [6] or no association [5, 7, 12, 41] with type 2 diabetes risk. Five cohort studies distinguished between lean and fatty fish: one showed a weak inverse association with fatty fish but not with lean fish [41], one observed higher risk associated with lean fish but not with fatty fish [7], one observed an inverse association with lean fish but not with fatty fish [14], and two did not observe statistically significant associations [6, 12].

Few previous studies have taken preparation methods into account. Only one study investigated fried fish consumption, showing no association comparing $\geq 1$ vs. $<1$ serving/week, whereas an inverse association with type 2 diabetes risk was observed for total fish [6]. In one other study, a direct association observed for total fish consumption was attenuated after further adjusting for intake of fried fish [7]. It is possible that different fish consumption habits and patterns in different populations, such as frequency of frying, have a part in explaining the heterogeneity among reports on the relationship between fish and diabetes. In our study population, $40 \%$ of the total fish consumed was fried. High consumption of fried foods in general has previously been reported to be associated with type 2 diabetes 
[42], as well as general and central obesity [43, 44], high blood pressure [44] and development of the metabolic syndrome [45]. In studies observing inverse associations between non-fried fish consumption and risk of heart failure [23, 26], ischaemic heart disease [22], stroke [24] and atrial fibrillation [25], high consumption of fried fish has been associated with higher risk or no association. Absorption of fat during frying results in more energy-dense foods and may contribute to a higher overall fat intake. The fatty acid composition of the food also changes, and frying fish may lead to a loss of long-chain omega-3 fatty acids and an increase in other fatty acids depending on the type of fat used for frying [46]. Moreover, high-temperature cooking, such as frying, induces formation of AGEs, which may contribute to insulin resistance [20]. Frying may further contribute to the formation of mutagenic compounds, such as heterocyclic amines [21]. We cannot, however, exclude that frying is a marker for other "unhealthy" behaviours that are unmeasured.

The present study is to our knowledge the first to report results for fish consumption taking dietary exposure to PCB and $\mathrm{MeHg}$ into account. Fish consumption and dietary contaminant exposure were highly correlated, which may inflate confidence intervals and bias the results [47]. However, adjustment for the contaminants did not markedly inflate confidence intervals. In addition, the large sample size of the study and fairly similar results across strata of dietary PCB and $\mathrm{MeHg}$ exposure further support that the adjustment did not produce spurious results in multivariable-adjusted model 2. In a recent meta-analysis of prospective studies on plasma or serum PCBs in relation to incident diabetes, higher concentration of total PCBs was associated with higher risk [29]. Results from prospective studies on toenail or hair mercury have suggested higher risk [30] or no association with diabetes [15, 48]. Given the regional variation of contaminant concentrations in seafood $[49,50]$, it is possible that adverse effects by PCBs, $\mathrm{MeHg}$ or other contaminants in part may explain the inconsistent findings reported on the relationship between fish consumption and risk of diabetes. Studies of PCB concentrations in serum or plasma have shown several-fold lower concentrations in populations from parts of Asia and Africa than in populations from parts of Europe and the USA [51]. The direct association observed for shellfish consumption was on the contrary not affected by adjustment for dietary $\mathrm{PCB}$ and $\mathrm{MeHg}$ exposure. A potential explanation for the association with shellfish could be related to how shellfish is consumed, i.e., in Sweden often with mayonnaise or other fatty condiments.

Potential benefits of fish consumption in relation to insulin resistance and type 2 diabetes have been suggested to be mediated through the content of long-chain n-3 fatty acids, based on their inhibitory effects on inflammatory pathways and activation of peroxisome proliferator-activated receptors [52]. Most randomized controlled trials examining the effects of long-chain n-3 fatty acid supplementation on insulin sensitivity have, however, found no effects [53], and as for fish consumption, observational findings on dietary intake of long-chain n-3 fatty acids have been mixed [17]. Fish is an important source of other nutrients that may modify the risk of type 2 diabetes, such as vitamin $\mathrm{D}$ and selenium. Observational evidence suggests that low vitamin D status is associated with higher risk of type 2 diabetes [54]. However, results from a large mendelian randomization analysis indicate that the association might not be causal [55] and evidence from randomized controlled trials does not support prevention of diabetes-related outcomes through vitamin D supplementation [56]. Higher toenail selenium was associated with lower risk in a recent large prospective study [57], whereas long-term selenium supplementation (high doses) has been associated with higher diabetes risk [58]. Further, intake of fish protein has been suggested to have beneficial effects on glucose tolerance and insulin sensitivity $[59,60]$.

Strengths of this study include its prospective population-based design, which precludes recall bias, as well as detailed dietary information and data on other lifestyle factors. Further strengths are the size of the study and the relatively large number of incident cases of type 2 diabetes. By linkage of our cohort to national registers using the individual personal registration number provided to all Swedish citizens, we were able to objectively ascertain type 2 diabetes cases and ensure comprehensive follow-up (not relying on response rates). However, because of the progressive nature of the disease with no clear onset, and the fact that type 2 diabetes rarely leads to hospitalization in the initial stages, it is likely that some early cases remain unregistered and thus were missed.

A limitation of our study is its observational design, leading to the possibility that the results may have been influenced by other habits and behaviours linked to both fish consumption and type 2 diabetes. Although we adjusted for a range of potential confounders, unmeasured or residual confounding cannot be entirely ruled out. Further, the dietary intake estimates were based on a selfreported FFQ, administered at a single time point with three single questions of fish, which inevitably leads to some degree of measurement error of the intake. Due to a larger within-food variation for contaminants as compared to intrinsic components in food, the precision of the dietary contaminant exposure estimates is further limited. Because exposure information was collected before the occurrence of the outcome, any misclassification would, however, be non-differential and most likely attenuate the association. Finally, the questionnaire design did not allow us to separately assess consumption of non-fried fish. 
In conclusion, these findings suggest that preparation methods may be important when considering the relationship between fish consumption and type 2 diabetes risk. We observed no overall association between total fish consumption and type 2 diabetes, whereas fried fish and shellfish consumption was associated with higher incidence. Our results further indicated that dietary exposure to environmental contaminants such as PCBs and $\mathrm{MeHg}$ may influence the net benefit of fish consumption. Current public health recommendations are not challenged on the basis of our results. However, general advice on regular fish consumption may be too imprecise, and advice on preparation methods as well as on specific fish species sub-types (varying in contamination) may be needed in order to benefit from increased consumption,

Acknowledgments This work was supported by research grants from the Swedish Research Council/Committee for Research Infrastructure for maintenance of the Cohort of Swedish Men, from the Karolinska Institutet's Award for PhD students (KID-funding) and from the Karolinska Institutet's Distinguished Professor Award. NGF acknowledges core support from the Medical Research Council Epidemiology Unit (MC_UU_12015/5). We would like to thank the NDR working group in Sweden as well as the local coordinators who contributed with registration of patient data.

\section{Compliance with ethical standards}

Conflict of interest The authors declare that they have no conflict of interest.

\section{References}

1. Whiting DR, Guariguata L, Weil C, Shaw J (2011) IDF diabetes atlas: global estimates of the prevalence of diabetes for 2011 and 2030. Diabetes Res Clin Pract 94:311-321

2. He K (2009) Fish, long-chain omega-3 polyunsaturated fatty acids and prevention of cardiovascular disease-Eat fish or take fish oil supplement? Prog Cardiovasc Dis 52:95-114

3. Montonen J, Jarvinen R, Heliovaara M, Reunanen A, Aromaa A, Knekt P (2005) Food consumption and the incidence of type II diabetes mellitus. Eur J Clin Nutr 59:441-448

4. Vang A, Singh PN, Lee JW, Haddad EH, Brinegar CH (2008) Meats, processed meats, obesity, weight gain and occurrence of diabetes among adults: findings from Adventist Health Studies. Ann Nutr Metab 52:96-104

5. Kaushik M, Mozaffarian D, Spiegelman D, Manson JE, Willett WC, Hu FB (2009) Long-chain omega-3 fatty acids, fish intake, and the risk of type 2 diabetes mellitus. Am J Clin Nutr 90:613-620

6. Patel PS, Sharp SJ, Luben RN, Khaw KT, Bingham SA, Wareham NJ, Forouhi NG (2009) Association between type of dietary fish and seafood intake and the risk of incident type 2 diabetes: the European prospective investigation of cancer (EPIC)-Norfolk cohort study. Diabetes Care 32:1857-1863

7. van Woudenbergh GJ, van Ballegooijen AJ, Kuijsten A, Sijbrands EJ, van Rooij FJ, Geleijnse JM, Hofman A, Witteman JC, Feskens EJ (2009) Eating fish and risk of type 2 diabetes: a population-based, prospective follow-up study. Diabetes Care 32:2021-2026
8. Patel PS, Sharp SJ, Jansen E, Luben RN, Khaw KT, Wareham NJ, Forouhi NG (2010) Fatty acids measured in plasma and erythrocyte-membrane phospholipids and derived by food-frequency questionnaire and the risk of new-onset type 2 diabetes: a pilot study in the European Prospective Investigation into Cancer and Nutrition (EPIC)-Norfolk cohort. Am J Clin Nutr 92:1214-1222

9. Brostow DP, Odegaard AO, Koh WP, Duval S, Gross MD, Yuan JM, Pereira MA (2011) Omega-3 fatty acids and incident type 2 diabetes: the Singapore Chinese Health Study. Am J Clin Nutr 94:520-526

10. Djousse L, Biggs ML, Lemaitre RN, King IB, Song X, Ix JH, Mukamal KJ, Siscovick DS, Mozaffarian D (2011) Plasma omega-3 fatty acids and incident diabetes in older adults. Am J Clin Nutr 94:527-533

11. Djousse L, Gaziano JM, Buring JE, Lee IM (2011) Dietary omega-3 fatty acids and fish consumption and risk of type 2 diabetes. Am J Clin Nutr 93:143-150

12. Nanri A, Mizoue T, Noda M, Takahashi Y, Matsushita Y, PoudelTandukar K, Kato M, Oba S, Inoue M, Tsugane S (2011) Fish intake and type 2 diabetes in Japanese men and women: the Japan Public Health Center-based Prospective Study. Am J Clin Nutr 94:884-891

13. Villegas R, Xiang YB, Elasy T, Li HL, Yang G, Cai H, Ye F, Gao YT, Shyr Y, Zheng W, Shu XO (2011) Fish, shellfish, and longchain n-3 fatty acid consumption and risk of incident type 2 diabetes in middle-aged Chinese men and women. Am J Clin Nutr 94:543-551

14. Rylander C, Sandanger TM, Engeset D, Lund E (2014) Consumption of lean fish reduces the risk of type 2 diabetes mellitus: a prospective population based cohort study of Norwegian women. PLoS ONE 9:e89845

15. Virtanen JK, Mursu J, Voutilainen S, Uusitupa M, Tuomainen TP (2014) Serum omega-3 polyunsaturated fatty acids and risk of incident type 2 diabetes in men: the Kuopio Ischaemic Heart Disease Risk Factor study. Diabetes Care 37:189-196

16. Ericson U, Sonestedt E, Gullberg B, Hellstrand S, Hindy G, Wirfalt E, Orho-Melander M (2013) High intakes of protein and processed meat associate with increased incidence of type 2 diabetes. Br J Nutr 109:1143-1153

17. Wallin A, Di Giuseppe D, Orsini N, Patel PS, Forouhi NG, Wolk A (2012) Fish consumption, dietary long-chain n-3 fatty acids, and risk of type 2 diabetes: systematic review and meta-analysis of prospective studies. Diabetes Care 35:918-929

18. Xun P, He K (2012) Fish consumption and incidence of diabetes: meta-analysis of data from 438,000 individuals in 12 independent prospective cohorts with an average 11-year follow-up. Diabetes Care 35:930-938

19. Wu JH, Micha R, Imamura F, Pan A, Biggs ML, Ajaz O, Djousse L, Hu FB, Mozaffarian D (2012) Omega-3 fatty acids and incident type 2 diabetes: a systematic review and meta-analysis. Br J Nutr 107(Suppl 2):S214-S227

20. Mark AB, Poulsen MW, Andersen S, Andersen JM, Bak MJ, Ritz C, Holst JJ, Nielsen J, de Courten B, Dragsted LO, Bugel SG (2014) Consumption of a diet low in advanced glycation end products for 4 weeks improves insulin sensitivity in overweight women. Diabetes Care 37:88-95

21. Felton JS, Malfatti MA, Knize MG, Salmon CP, Hopmans EC, Wu RW (1997) Health risks of heterocyclic amines. Mutat Res 376:37-41

22. Mozaffarian D (2003) Cardiac benefits of fish consumption may depend on the type of fish meal consumed: the cardiovascular health study. Circulation 107:1372-1377

23. Mozaffarian D, Bryson CL, Lemaitre RN, Burke GL, Siscovick DS (2005) Fish intake and risk of incident heart failure. J Am Coll Cardiol 45:2015-2021 
24. Mozaffarian D, Longstreth WT Jr, Lemaitre RN, Manolio TA, Kuller LH, Burke GL, Siscovick DS (2005) Fish consumption and stroke risk in elderly individuals: the cardiovascular health study. Arch Intern Med 165:200-206

25. Mozaffarian D (2004) Fish intake and risk of incident atrial fibrillation. Circulation 110:368-373

26. Belin RJ, Greenland P, Martin L, Oberman A, Tinker L, Robinson J, Larson J, Van Horn L, Lloyd-Jones D (2011) Fish intake and the risk of incident heart failure: the women's health initiative. Circ Heart Fail 4:404-413

27. Bergkvist C, Akesson A, Glynn A, Michaelsson K, Rantakokko P, Kiviranta H, Wolk A, Berglund M (2012) Validation of questionnaire-based long-term dietary exposure to polychlorinated biphenyls using biomarkers. Mol Nutr Food Res $56: 1748-1754$

28. Strom S, Helmfrid I, Glynn A, Berglund M (2011) Nutritional and toxicological aspects of seafood consumption-an integrated exposure and risk assessment of methylmercury and polyunsaturated fatty acids. Environ Res 111:274-280

29. Wu H, Bertrand KA, Choi AL, Hu FB, Laden F, Grandjean P, Sun Q (2013) Persistent organic pollutants and type 2 diabetes: a prospective analysis in the nurses' health study and meta-analysis. Environ Health Perspect 121:153-161

30. He K, Xun P, Liu K, Morris S, Reis J, Guallar E (2013) Mercury exposure in young adulthood and incidence of diabetes later in life: the CARDIA Trace Element Study. Diabetes Care 36:1584-1589

31. Norman A, Bellocco R, Vaida F, Wolk A (2002) Total physical activity in relation to age, body mass, health and other factors in a Cohort of Swedish Men. Int J Obes Relat Metab Disord 26:670-675

32. Hansson LM, Galanti MR (2000) Diet-associated risks of disease and self-reported food consumption: How shall we treat partial nonresponse in a food frequency questionnaire? Nutr Cancer 36:1-6

33. Bergström L, Kylberg E, Hagman U, Erikson H, Bruce A (1991) The food composition database KOST: the National Administration's information system for nutritive values of food. Vår Föda 43:439-447

34. Messerer M, Johansson SE, Wolk A (2004) The validity of questionnaire-based micronutrient intake estimates is increased by including dietary supplement use in Swedish Men. J Nutr 134:1800-1805

35. Fung TT, Chiuve SE, McCullough ML, Rexrode KM, Logroscino G, Hu FB (2008) Adherence to a DASH-style diet and risk of coronary heart disease and stroke in women. Arch Intern Med 168:713-720

36. Glynn AW, Granath F, Aune M, Atuma S, Darnerud PO, Bjerselius R, Vainio H, Weiderpass E (2003) Organochlorines in Swedish women: determinants of serum concentrations. Environ Health Perspect 111:349-355

37. Pauwels A, Covaci A, Weyler J, Delbeke L, Dhont M, De Sutter P, D'Hooghe T, Schepens PJ (2000) Comparison of persistent organic pollutant residues in serum and adipose tissue in a female population in Belgium, 1996-1998. Arch Environ Contam Toxicol 39:265-270

38. Willett W, Stampfer MJ (1986) Total energy intake: implications for epidemiologic analyses. Am J Epidemiol 124:17-27

39. Norman A, Bellocco R, Bergstrom A, Wolk A (2001) Validity and reproducibility of self-reported total physical activity-differences by relative weight. Int J Obes Relat Metab Disord 25:682-688

40. Swedish National Diabetes Register (NDR)-Centre of Registers, Region Västra Götaland (2014). Annual Report 2013: Results. https://www.ndr.nu/pdfs/Annual_Report_NDR_2013. pdf. Accessed 11 Nov 2015
41. Patel PS, Forouhi NG, Kuijsten A, Schulze MB, van Woudenbergh GJ, Ardanaz E, Amiano P, Arriola L, Balkau B, Barricarte A, Beulens JW, Boeing H, Buijsse B, Crowe FL, de LauzonGuillan B, Fagherazzi G, Franks PW, Gonzalez C, Grioni S, Halkjaer J, Huerta JM, Key TJ, Kuhn T, Masala G, Nilsson P, Overvad K, Panico S, Quiros JR, Rolandsson O, Sacerdote C, Sanchez MJ, Schmidt EB, Slimani N, Spijkerman AM, Teucher $\mathrm{B}$, Tjonneland A, Tormo MJ, Tumino R, van der AD, van der Schouw YT, Sharp SJ, Langenberg C, Feskens EJ, Riboli E, Wareham NJ (2012) The prospective association between total and type of fish intake and type 2 diabetes in 8 European countries: EPIC-InterAct Study. Am J Clin Nutr 95:1445-1453

42. Cahill LE, Pan A, Chiuve SE, Sun Q, Willett WC, Hu FB, Rimm EB (2014) Fried-food consumption and risk of type 2 diabetes and coronary artery disease: a prospective study in 2 cohorts of US women and men. Am J Clin Nutr 100:667-675

43. Guallar-Castillon P, Rodriguez-Artalejo F, Fornes NS, Banegas JR, Etxezarreta PA, Ardanaz E, Barricarte A, Chirlaque MD, Iraeta MD, Larranaga NL, Losada A, Mendez M, Martinez C, Quiros JR, Navarro C, Jakszyn P, Sanchez MJ, Tormo MJ, Gonzalez CA (2007) Intake of fried foods is associated with obesity in the cohort of Spanish adults from the European Prospective Investigation into Cancer and Nutrition. Am J Clin Nutr 86:198-205

44. Sayon-Orea C, Martinez-Gonzalez MA, Gea A, Flores-Gomez E, Basterra-Gortari FJ, Bes-Rastrollo M (2014) Consumption of fried foods and risk of metabolic syndrome: the SUN cohort study. Clin Nutr 33:545-549

45. Lutsey PL, Steffen LM, Stevens J (2008) Dietary intake and the development of the metabolic syndrome: the Atherosclerosis Risk in Communities study. Circulation 117:754-761

46. Wagner KH, Elmadfa I (2012) Chemical and biological modulations of food due to the frying process. Int J Vitam Nutr Res $82: 163-167$

47. Elmstahl S, Gullberg B (1997) Bias in diet assessment methodsconsequences of collinearity and measurement errors on power and observed relative risks. Int J Epidemiol 26:1071-1079

48. Mozaffarian D, Shi P, Morris JS, Grandjean P, Siscovick DS, Spiegelman D, Hu FB (2013) Methylmercury exposure and incident diabetes in U.S. men and women in two prospective cohorts. Diabetes Care 36:3578-3584

49. Sunderland EM (2007) Mercury exposure from domestic and imported estuarine and marine fish in the U.S. seafood market. Environ Health Perspect 115:235-242

50. Aguilar A, Borrell A, Reijnders PJ (2002) Geographical and temporal variation in levels of organochlorine contaminants in marine mammals. Mar Environ Res 53:425-452

51. Glynn AW, Wolk A, Aune M, Atuma S, Zettermark S, MaehleSchmid M, Darnerud PO, Becker W, Vessby B, Adami HO (2000) Serum concentrations of organochlorines in men: a search for markers of exposure. Sci Total Environ 263:197-208

52. Deckelbaum RJ, Worgall TS, Seo T (2006) n-3 fatty acids and gene expression. Am J Clin Nutr 83:1520S-1525S

53. Akinkuolie AO, Ngwa JS, Meigs JB, Djousse L (2011) Omega-3 polyunsaturated fatty acid and insulin sensitivity: a meta-analysis of randomized controlled trials. Clin Nutr 30:702-707

54. Afzal S, Bojesen SE, Nordestgaard BG (2013) Low 25-hydroxyvitamin D and risk of type 2 diabetes: a prospective cohort study and metaanalysis. Clin Chem 59:381-391

55. Ye Z, Sharp SJ, Burgess S, Scott RA, Imamura F, Langenberg C, Wareham NJ, Forouhi NG (2015) Association between circulating 25-hydroxyvitamin $\mathrm{D}$ and incident type 2 diabetes: a mendelian randomisation study. Lancet Diabetes Endocrinol 3:35-42

56. Seida JC, Mitri J, Colmers IN, Majumdar SR, Davidson MB, Edwards AL, Hanley DA, Pittas AG, Tjosvold L, Johnson JA (2014) Effect of vitamin D supplementation on 
improving glucose homeostasis and preventing diabetes: a systematic review and meta-analysis. J Clin Endocrinol Metab 99:3551-3560

57. Park K, Rimm EB, Siscovick DS, Spiegelman D, Manson JE, Morris JS, Hu FB, Mozaffarian D (2012) Toenail selenium and incidence of type 2 diabetes in U.S. men and women. Diabetes Care 35:1544-1551

58. Stranges S, Marshall JR, Natarajan R, Donahue RP, Trevisan M, Combs GF, Cappuccio FP, Ceriello A, Reid ME (2007) Effects of long-term selenium supplementation on the incidence of type 2 diabetes: a randomized trial. Ann Intern Med 147:217-223
59. Vikoren LA, Nygard OK, Lied E, Rostrup E, Gudbrandsen OA (2013) A randomised study on the effects of fish protein supplement on glucose tolerance, lipids and body composition in overweight adults. Br J Nutr 109:648-657

60. Ouellet V, Marois J, Weisnagel SJ, Jacques H (2007) Dietary cod protein improves insulin sensitivity in insulin-resistant men and women: a randomized controlled trial. Diabetes Care 30:2816-2821 